九州大学学術情報リポジトリ

Kyushu University Institutional Repository

\title{
Optimum Design of a Passive Suspension system of a Semi-Submersible for Pitching Reduction
}

\section{Zhu, Hongzhong}

Research and Education Center for Advanced Energy Materials, Devices, and Systems, Kyushu University

$\mathrm{Hu}$, Changhong

Research Institute for Applied Mechanics, Kyushu University

Liu, Yingyi

Research Institute for Applied Mechanics, Kyushu University

http://hdl. handle. net/2324/4055229

出版情報: Journal of Dynamic Systems, Measurement, and Control. 138 (12), pp. 121003-，2016-12. The American Society of Mechanical Engineers

バージョン :

権利関係：○ ASME 


\section{American Society of Mechanical Engineers}

SETTING THE STANDARD

\section{ASME Accepted Manuscript Repository}

\section{Institutional Repository Cover Sheet}

Repository Section, Kyushu University Library, Japan

ASME Paper Title: Optimum Design of a Passive Suspension System of a Semisubmersible for Pitching Reduction

Authors: Hongzhong Zhu, Changhong Hu, Yingyi Liu

ASME Journal Title: Journal of Dynamic Systems, Measurement, and Control

Volume/Issue v.138 issue. 12

Date of Publication (VOR* Online)

August 11, 2016

https://asmedigitalcollection.asme.org/dynamicsystems/article-

abstract/138/12/121003/472920/Optimum-Design-of-a-Passive-Suspension-System-of-

ASME Digital Collection URL: a?redirectedFrom=fulltext

DOI: $\quad$ https://doi.org/10.1115/1.4033948

*VOR (version of record) 


\section{Optimum Design of a Passive Suspension system of a Semi-Submersible for Pitching Reduction}

\author{
Hongzhong Zhu* \\ Research and Education Center for \\ Advanced Energy Materials, Devices, \\ and Systems, Kyushu University \\ Kasuga 816-8580, Japan \\ Email: zhuhongzhong@riam.kyushu-u.ac.jp
}

\author{
Changhong $\mathrm{Hu}$ \\ Research Institute for Applied \\ Mechanics, Kyushu University \\ Kasuga 816-8580, Japan \\ hu@riam.kyushu-u.ac.jp
}

\author{
Yingyi Liu \\ Research Institute for Applied \\ Mechanics, Kyushu University \\ Kasuga 816-8580, Japan \\ liuyingyi@riam.kyushu-u.ac.jp
}

With the development of ocean energy exploration, reliable semi-submersible platforms with very small motion are expected to develop. Especially, in a floating offshore wind turbine system, the maximum pitching amplitude is required to be less than a few degrees. To reduce wave-induced pitch motion, a new type semi-submersible with suspensions and a design method of the suspension coefficients are presented. In practical case, an add-on wave energy dissipation device mounted on a floating platform, such as the combined wavewind energy converter system, could be regarded as the suspension system. In this study, firstly, the conceptual semisubmersible is described. Then, the hydrodynamic loads to the semi-submersible are linearized so that the whole system is expressed by a state-space model. The suspension design problem is transformed into solving a constrained $\mathbf{H}_{\infty}$ optimization problem, which after all is the optimal controller design of a feedback system. Finally, numerical examples are performed to verify the effectiveness of the design. The results illustrate that the pitch motion of the semi-submersible can be remarkably reduced by the designed suspensions.

\section{Introduction}

A semi-submersible platform is a specialised marine vessel used in a number of offshore applications such as offshore drilling rigs and heavy lift cranes. As the development of offshore wind energy exploration, reliable and low cost semi-submersible platforms are increasingly required [1]. Although the platforms are designed with good sea-keeping performance and good stability, environmental

* Address all correspondence to this author. forces induced by wind, waves and ocean currents can, however, induce undesired heave and pitch motion, which would remarkably reduce the fatigue life of devices on the platforms [2-4]. Suzuki and Sato (2007) have performed some pioneering work on investigating the effects of motion of floating platform on the strength of offshore wind turbine blades, and they come to the result that pitching with an amplitude of 5 degrees will lead to a $50 \%$ inclease of sectional modulus of a blade to avoid fatigue failure [5].

In the last decade there have been some floating platforms built for full-scale experimentation like GustosMSC Tri-Floater [6], WindFloat [7], and the V-shape semisubmersible floater [8]. Most of them are designed with three or four rigidly-connected columns having a small waterplane area to decrease the wave effects. Even by sophisticated analysis and design, the maximum inclination of a floater would reach to 10 degrees according to model tests [6]. In order to further reduce the undesired motion, various methods and state-of-art structures were proposed in the literature. In $[9,10]$, novel water-entrapment plates with large horizontal skirts to increase the added-mass and viscous loads were designed to semi-submersibles. In the methods, the natural periods of the floaters in heave, pitch and roll can be adjusted to avoid the resonance with environmental forces. Though the water-entrapment plates can be systematically designed considering strength analysis and fatigue loads, the fabrication cost and maintenance, however, would limit its applications. In addition, Roddier and Cermelli (2013) have proposed an interesting concept that the floater is composed of three column tubes which are partly filled with water [11]. The water can be pumped between the three columns to balance out the environment forces so that the inclination of 
platform could be controlled. A major advantage of the concept is that the pitch motion can be controlled by pumping the water from some columns to the others. However, the response may be too slow to wind turbulence and non-regular waves.

Recent studies also focus on combining wave energy converters (WEC) with floating offshore wind turbine (FOWT) for a better utilization of marine space and lower installation cost $[12,13]$. Though there are many advantages, the combination also introduces the inter-influence between WEC and FOWT, which makes the whole system complex. Perez and Iglesias (2012) reported that particular attention should be paid to the wind turbine foundation affected by the installation of the WEC since the inclination of the foundation may exceed the design limit [14]. Hence, to optimally design the combination system becomes an urgent work.

In this paper, a conceptual design of a semi-submersible with passive suspensions is presented. The system consists of a platform and some vertical columns working as the wave energy dissipation devices. The columns are mounted to the platform via suspensions modeled with springs and dampers. The columns together with the suspensions can also be regarded as the WECs in which the dampers are regarded as the power take-off devices. The purpose of this study is to minimize the pitch motion of the platform by designing the suspensions under some given sea state. Though the concept is similar to the suspension design of land-based vehicles, the wave-structure interaction makes the design much more complicated than the later case.

The remainder of this paper is organized as follows. Firstly, the concept of the semi-submersible platform is introduced. Secondly, the mathematical models of the platform are presented. Then, a method to design the mass of the columns, the spring and damper coefficients is proposed. Some numerical examples are finally given to demonstrate the effectiveness of the design method.

\section{Semi-Submersible and Problem Description}

The schematic of the proposed semi-submersible is shown in Fig. 1. As the first step of the research, a twodimensional model which is bilaterally symmetric is considered for simplicity. The platform is supported by the submerged pontoon via the pillars and the vertical columns located at the both ends via suspensions. Without loss of generality, the suspension is modeled with the combination of a linear spring $k$ and a linear damper $c$. For analysis, the amplitude of motion is assumed to be small and there is enough space between the columns and the main body to avoid the mechanical crash within the components. In the figure, $L$ is the distance between the two columns, $z_{1}$ and $z_{2}$ are the displacements of column 1 and column 2, $\theta$ is the pitching angle of the main body, and $z_{p_{1}}$ and $z_{p_{2}}$ are the displacement of the platform in vertical direction at the ends. Note that the system can also be regarded as a floating wave energy converter where the dampers $c$ is regarded as the power take-off devices.

This work focus on reducing the wave induced motion

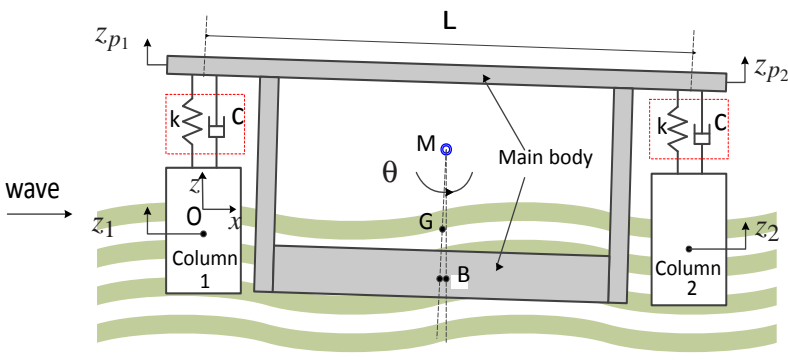

Fig. 1. Schematic of conceptual design.

by optimally designing the mass of the columns and the suspension coefficients without considering the effect of wind. Although large static inclination of a semi-submersible may be induced by the thrust of wind, the wind-induced fatigue would be much smaller than the wave-induced fatigue since the variation of wind forces is relatively low and long term.

\section{Mathematical Models}

\subsection{Modelling of Semi-Submersible}

Considering small amplitude of motion and negligible viscous effects, the motion equations of the system can be expressed as

$$
\begin{aligned}
J_{p} \ddot{\theta} & =\tau_{\mathrm{rad}}+\tau_{\mathrm{res}}+\tau_{\mathrm{exc}}+\frac{L}{2}\left(f_{1}-f_{2}\right), \\
m_{p} \ddot{z}_{o} & =F_{\mathrm{rad}_{o}}+F_{\mathrm{res}_{o}}+F_{\mathrm{exc}_{o}}+f_{1}+f_{2}, \\
m_{c} \ddot{z}_{1} & =F_{\mathrm{rad}_{1}}+F_{\mathrm{res}_{1}}+F_{\mathrm{exc}_{1}}-f_{1}, \\
m_{c} \ddot{z}_{2} & =F_{\mathrm{rad}_{2}}+F_{\mathrm{res}_{2}}+F_{\mathrm{exc}_{2}}-f_{2},
\end{aligned}
$$

where $m_{p}$ is the mass of the main body, $J_{p}$ is the moment of inertia of the main body in pitch direction, $m_{c}$ is the mass of one column, $\tau_{\text {rad }}, F_{\text {rad }_{o}}, F_{\text {rad }_{1}}, F_{\mathrm{rad}_{2}}$ are the radiation loads arising from the change in momentum of the fluid due to the motion of corresponding components, $\tau_{\text {res }}, F_{\text {res }_{o}}, F_{\text {res }_{1}}, F_{\text {res }_{2}}$ are the restoring loads due the gravity and buoyancy, and $\tau_{\mathrm{exc}}, F_{\mathrm{exc}_{o}}, F_{\mathrm{exc}_{1}}, F_{\mathrm{exc}_{2}}$ are the wave excitation loads consisting of Froude-Krylov forces and diffraction forces. The radiation loads and the wave excitation loads can be calculated by potential flow method assuming the fluid to be inviscid, incompressible, and irrotational. Analysis of hydrodynamic interaction between the different bodies is also available by many (commercial) source [15]. The terms $f_{1}, f_{2}$ are the counteracting forces from the suspensions that

$$
\begin{aligned}
& f_{1}=k z_{r_{1}}+c \dot{z}_{r_{1}}, \\
& f_{2}=k z_{r_{1}}+c \dot{z}_{r_{1}} .
\end{aligned}
$$

Here, $z_{r_{1}} \doteq z_{1}-z_{p_{1}}, z_{r_{2}} \doteq z_{2}-z_{p_{2}}$ are the relative displacements between the main body and the columns.

Note that the pitch motion of the columns is not considered in the models expressed by (1-4). In practical case, the columns should be designed with high restoring capability in pitch direction while keeping the pitch natural frequency 
far away from the wave band. Besides, the hydrodynamic horizontal forces on the columns and their effect on the pitch motion of the platform are ignored since the arm of force of the horizontal forces is usually small compared to that of the vertical forces. If this is not the case, pitch motion equations of the columns should also be considered in the models.

In order to evaluate the effect of mass distribution on pitch motion of the platform, the mass ratio defined by $a:=\frac{m_{c}}{m_{p}}$ is introduced, and the following two conditions are considered:

$$
\left\{\begin{array}{l}
2 m_{c}+m_{p}=M_{\mathrm{all}}=\mathrm{constant} \\
J_{p} \propto m_{p}
\end{array}\right.
$$

where $M_{\text {all }}$ is the total mass. For the sake of convenience, the waterplane area of the main body is set to be much smaller than that of the columns. Under this assumption, the first condition in (7) indicates that both the draft of main body and that of columns are unaltered with $a$, though the static load of the suspensions varies accordingly. Hence, the hydrodynamic coefficients of the system including the added mass, potential damping and the impulse response functions of wave excitation loads are insensitive to $a$. The second condition shows that the moment of inertia $J_{p}$ is set directly linear to the mass $m_{p}$. More accurate relation can also be given for practical cases. By calculation, $m_{c}, m_{p}$ and $J_{p}$ satisfy

$$
\begin{aligned}
& m_{c}=\frac{a}{1+2 a} M_{\text {all }} \\
& m_{p}=\frac{1}{1+2 a} M_{\text {all }} \\
& J_{p}=\gamma \frac{1}{1+2 a} M_{\mathrm{all}},
\end{aligned}
$$

where $\gamma$ is a given coefficient.

According to [16], the radiation load of pitching motion in (1) can be expressed by

$$
\tau_{\mathrm{rad}}=-J_{\infty} \ddot{\theta}-\int_{0}^{t} K_{\theta_{r}}(t-\tau) \dot{\theta}(\tau) d \tau
$$

where the first term owes to the acceleration of the platform and $J_{\infty}$ is a constant positive added moment of inertia. The second term represents fluid-memory model that capture the energy transfer from the motion of platform to the radiated waves. The kernel of the convolution term, $K_{\theta_{r}}(t)$, which is known as retardation or memory function, is the impulse response function of the radiation in pitch direction. The convolution integral term can be approximated by a linear subsystem using state-space model

$$
\left\{\begin{array}{l}
\dot{x}_{\theta_{r}}(t)=A_{\theta_{r}} x_{\theta_{r}}(t)+B_{\theta_{r}} \dot{\theta}(t), \\
y_{\theta_{r}}(t)=C_{\theta_{r}} x_{\theta_{r}}(t) \approx \int_{0}^{t} K_{\theta_{r}}(t-\tau) \dot{\theta}(\tau) d \tau,
\end{array}\right.
$$

where $x_{\theta_{r}} \in \mathcal{R}^{n \times 1}$ is state vector, and $A_{\theta_{r}}, B_{\theta_{r}}, C_{\theta_{r}}$ are the system matrices that can be obtained from theory or experiments by system identification [17].

By combining the equations (1), (11) and (12), and applying the linearized restoring force

$$
\tau_{\text {res }}=-\rho g V \overline{\mathrm{GM}} \theta
$$

where $\rho$ is the density of water, $g$ is the gravitational acceleration, $V$ is the displaced volume of water and $\overline{\mathrm{GM}}$ is the meta-centric height, the pitch motion equation of the main platform (1) becomes

$$
\mathcal{G}_{\theta}:\left\{\begin{array}{l}
\dot{x}_{\theta}(t)=A_{\theta} x_{\theta}(t)+B_{\theta_{a}} \tau_{\mathrm{exc}}(t)+B_{\theta_{b}} u_{f}(t), \\
y_{\theta}(t)=C_{\theta} x_{\theta}(t) .
\end{array}\right.
$$

where $y_{\theta}=[\theta, \dot{\theta}]^{T}$ is the output vector, $x_{\theta}=\left[x_{\theta_{r}}^{T}, \theta, \dot{\theta}\right]^{T}$ is the state vector, $u_{f}=\left[f_{1}, f_{2}\right]^{T}$ is the force vector from (5) (6), and the matrices are

$$
\begin{aligned}
& A_{\theta}= {\left[\begin{array}{ccc}
A_{\theta_{r}} & 0_{n \times 1} & B_{\theta_{r}} \\
0_{1 \times n} & 0 & 1 \\
-\frac{C_{\theta_{r}}}{J_{p}(a)+J_{\infty}} & -\frac{\rho g V \overline{\mathrm{GM}}}{J_{p}(a)+J_{\infty}} & 0
\end{array}\right], } \\
& B_{\theta_{a}}= {\left[\begin{array}{c}
0_{n \times 1} \\
0 \\
\frac{1}{J_{p}(a)+J_{\infty}}
\end{array}\right], } \\
& B_{\theta_{b}}=\left[\begin{array}{cc}
0_{n \times 1} & 0_{n \times 1} \\
\frac{L}{2} \frac{1}{J_{p}(a)+J_{\infty}}-\frac{L}{2} \frac{1}{J_{p}(a)+J_{\infty}}
\end{array}\right], \\
& C_{\theta}=\left[\begin{array}{lll}
0_{1 \times n} & 1 & 0 \\
0_{1 \times n} & 0 & 1
\end{array}\right] .
\end{aligned}
$$

Here, $0_{i \times j}$ is the zero matrix with $i$ rows and $j$ columns.

Similarly, the motion equations (2), (3) and (4) can be expressed by

$$
\begin{gathered}
\mathcal{G}_{o}:\left\{\begin{array}{l}
\dot{x}_{o}(t)=A_{o} x_{o}(t)+B_{o_{a}} F_{\mathrm{exc}_{o}}(t)+B_{o_{b}} u_{f}(t) \\
y_{o}(t)=C_{o} x_{o}(t),
\end{array}\right. \\
\mathcal{G}_{c 1}:\left\{\begin{array}{l}
\dot{x}_{c 1}(t)=A_{c} x_{c 1}(t)+B_{c_{a}} F_{\mathrm{exc}_{1}}(t)+B_{c_{b}} f_{1}(t) \\
y_{c 1}(t)=C_{c} x_{c 1}(t),
\end{array}\right. \\
\mathcal{G}_{c 2}:\left\{\begin{array}{l}
\dot{x}_{c 2}(t)=A_{c} x_{c 2}(t)+B_{c_{a}} F_{\mathrm{exc}_{2}}(t)+B_{c_{b}} f_{2}(t) \\
y_{c 2}(t)=C_{c} x_{c 2}(t)
\end{array}\right.
\end{gathered}
$$

where $x_{o}, x_{c 1}$ and $x_{c 2}$ are state vectors, $y_{o}=\left[z_{o}, \dot{z}_{o}\right]^{T}, y_{c 1}=$ $\left[z_{1}, \dot{z}_{1}\right]^{T}$ and $y_{c 2}=\left[z_{2}, \dot{z}_{2}\right]^{T}$ are output vectors related to the positions and velocities, The matrices $\left(A_{o}, B_{o_{a}}, B_{o_{b}}, C_{o}\right)$ and $\left(A_{c}, B_{c_{a}}, B_{c_{b}}, C_{c}\right)$ can be derived by the same approach as aforementioned. 


\subsection{Modelling of Wave Excitation Loads}

According to linear theory, the excitation force is proportional to the incident wave elevation such that

$$
f_{e}(t)=\int_{-\infty}^{\infty} h_{f}(t-\tau) \eta(0, \tau) d \tau
$$

The term $\eta(x, t)$ is the incident wave elevation where $x$ is the horizontal coordinate in the direction of wave propagation, and $\eta(0, t)$ is the wave elevation at the origin. The term $h_{f}(t)$ is the impulse response function of excitation force coefficient which can be calculated by potential theory. For simplicity, the origin of wave is assumed to be located at the vertical axis through the centre of the Column 1. The following linear model is employed to approximate the excitation force $F_{\mathrm{exc}_{1}}$ :

$$
\mathcal{F}_{e_{1}}(s)=\frac{b_{c_{0}} s^{r}+b_{c_{1}} s^{r-1}+\cdots+b_{c_{r}}}{s^{r}+a_{c_{1}} s^{r-1}+\cdots+a_{c_{r}}} \cdot \pi(0, s),
$$

where $s$ is the Laplace operator, $\mathcal{F}_{e 1}$ are the Laplace transform of $F_{\mathrm{exc}_{1}}$. The term $\pi(0, s)$ is the Laplace transform of the wave $\eta(0, t)$. The coefficients $b_{c_{0}}, b_{c_{1}}, \cdots, b_{c_{r}}$ and $a_{c_{0}}, a_{c_{1}}, \cdots, a_{c_{r}}$ are the variables to be tuned to fit the real system via system identification, see [18] for comprehensive overviews. Similarly, the excitation forces $F_{\operatorname{exc}_{o}}, \tau_{\mathrm{exc}}$, and $F_{\mathrm{exc}_{2}}$ can be modeled as

$$
\begin{gathered}
\mathcal{F}_{e_{o}}(s)=\frac{b_{o_{0}} s^{r}+b_{o_{1}} s^{r-1}+\cdots+b_{o_{r}}}{s^{r}+a_{o_{1}} s^{r-1}+\cdots+a_{o_{r}}} \cdot \pi\left(\frac{L}{2}, s\right), \\
\mathcal{T}_{e}(s)=\frac{b_{\tau_{0}} s^{r}+b_{\tau_{1}} s^{r-1}+\cdots+b_{\tau_{r}}}{s^{r}+a_{\tau_{1}} s^{r-1}+\cdots+a_{\tau_{r}}} \cdot \pi\left(\frac{L}{2}, s\right), \\
\mathcal{F}_{e_{2}}(s)=\frac{b_{c_{0}} s^{r}+b_{c_{1}} s^{r-1}+\cdots+b_{c_{r}}}{s^{r}+a_{c_{1}} s^{r-1}+\cdots+a_{c_{r}}} \cdot \pi(L, s),
\end{gathered}
$$

where $\pi(d, s)$ ( $d=\frac{L}{2}$ or $\left.L\right)$ is the Laplace transform of wave $\eta(d, t)$. The term $\pi(d, s)$ can be regarded as a delayed wave function of $\pi(0, s)$, and it therefore can be approximated as $\pi(d, s) \approx e^{-T_{d} s} \pi(0, s)$ in which $T_{d}$ is the wave propagation period from the origin $x=0$ to a certain distance $d$. The first order Padé-approximation can be applied for linearizing this delayed term. In the case of irregular incident wave, the dominant wave frequency can be applied to calculate these periods. Accordingly, the models (19-22) can be linearized as

$$
\begin{aligned}
& \mathcal{H}_{F_{e_{1} 1} \leftarrow \eta}: \frac{\mathcal{F}_{e_{1}}(s)}{\pi(0, s)}=\left[\begin{array}{c|c}
A_{F_{e_{1}} \eta} & B_{F_{e_{1}} \eta} \\
\hline C_{F_{e_{1}} \eta} & D_{F_{e_{1}}} \eta
\end{array}\right], \\
& \mathcal{H}_{F_{\text {exco }} \leftarrow \eta}: \frac{\mathcal{F}_{e_{o}}(s)}{\pi(0, s)}=\left[\begin{array}{c|c}
A_{F_{e_{o}}} \eta & B_{F_{e_{o}} \eta} \\
\hline C_{F_{e_{o}}} \eta & D_{F_{e_{o}}} \eta
\end{array}\right], \\
& \mathcal{H}_{\text {Texc }_{e} \leftarrow \eta}: \frac{\mathcal{T}_{e}(s)}{\pi(0, s)}=\left[\begin{array}{c|c}
A_{T_{e} \eta} & B_{T_{e} \eta} \\
\hline C_{T_{e} \eta} & D_{T_{e} \eta}
\end{array}\right],
\end{aligned}
$$

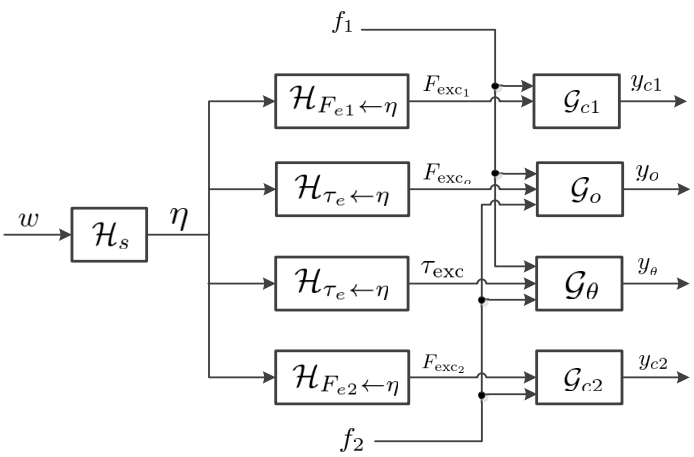

Fig. 2. Block diagram of the whole model.

and

$$
\mathcal{H}_{F_{\text {exc }} \leftarrow \eta}: \frac{\mathcal{F}_{e_{2}}(s)}{\pi(0, s)}=\left[\begin{array}{c|c}
A_{F_{e_{2}} \eta} & B_{F_{e_{2}} \eta} \\
\hline C_{F_{e_{2}} \eta} & D_{F_{e_{2}}} \eta
\end{array}\right],
$$

where the notation

$$
\left[\begin{array}{l|l}
\mathcal{A} & \mathcal{B} \\
\hline \mathcal{C} & \mathcal{D}
\end{array}\right]:=\mathcal{C}(s I-\mathcal{A})^{-1} \mathcal{B}+\mathcal{D}
$$

is used. The matrices in (23-26) can be calculated from the coefficients in (19-22).

Fig. 2 shows the integration of the models given by (1417) and (23-26). In the figure, in order to apply linear control theories for synthesis, a linear approximation of wave spectral density function, which is denoted as $\mathcal{H}_{s}$, is also introduced. The signal $w$ in the figure is a zero-mean Gaussian white noise process with unit power. In this study, a widely used second-order system is employed to approximate the wave spectral density function [19]:

$$
\mathcal{H}_{s}(s)=\frac{2 \lambda \omega_{0} \sigma s}{s^{2}+2 \lambda \omega_{0} s+\omega_{0}^{2}}
$$

Here, $\lambda$ is a damping coefficient, $\sigma$ is a constant describing the wave intensity and $\omega_{0}$ is the dominating wave frequency. A corresponding linear state-space model is

$$
\begin{aligned}
& \dot{x}_{w}(t)=A_{w} x_{w}(t)+B_{w} w(t) \\
& \eta(0, t)=C_{w} x_{w}(t)
\end{aligned}
$$

where $x_{w}$ is the state vector. The matrices are expressed by

$$
\begin{aligned}
A_{w} & =\left[\begin{array}{ll}
0 & 1 \\
-\omega_{0}^{2} & -2 \lambda \omega_{0}
\end{array}\right], B_{w}=\left[\begin{array}{l}
0 \\
2 \lambda \omega_{0} \sigma
\end{array}\right] \\
C_{w} & =\left[\begin{array}{ll}
0 & 1
\end{array}\right]
\end{aligned}
$$

In the next section, we will show that the optimal design of suspensions leads to a feedback controller design problem. 


\section{Design}

Integrating together the models (14-17), (23-26) and (29), the system can be expressed by

$$
\begin{aligned}
& \dot{x}(t)=A(a) x(t)+B_{1} w(t)+B_{2}(a) u_{f}(t), \\
& p(t)=C_{1} x(t), \\
& y(t)=C_{2} x(t),
\end{aligned}
$$

where $x=\left[x_{c 1}^{T}, x_{a}^{T}, x_{\theta}^{T}, x_{b}^{T}, x_{c 2}^{T}, x_{c}^{T}, x_{w}^{T}\right]^{T}$ is the state vector where $x_{a}, x_{b}$ and $x_{c}$ are the state vectors of subsystems (23), (25) and (26), respectively. The term $p=\theta$ is the performance output signal to be regulated, $y=\left[z_{r_{1}}, \dot{z}_{r_{1}}, z_{r_{2}}, \dot{z}_{r_{2}}\right]^{T}$ is the output vector of the relative motion between the main body and the columns. The inputs are the zero-mean Gaussian white noise $w$ and the force vector $u_{f}=\left[f_{1}, f_{2}\right]^{T}$. The matrices are given in (31) where $p=n+r$.

According to (5) and (6), we can obtain that

$$
u_{f}=\left[\begin{array}{l}
f_{1} \\
f_{2}
\end{array}\right]=K y
$$

where

$$
K=\left[\begin{array}{llll}
k & c & 0 & 0 \\
0 & 0 & k & c
\end{array}\right]
$$

Obviously, the design of suspension coefficients equals to determine the output feedback controller $K$ for the system (30). The relationship between the inputs and the outputs is shown in Fig. 3. The transfer matrix from the input $w$ to the performance output $\theta$, which is defined by $\mathcal{H}_{\theta w}$, is expressed by

$$
\mathcal{H}_{\theta w}(s)=C_{1}(s I-\tilde{A})^{-1} B_{1},
$$

where $I$ is identity matrix with appropriate dimension and $\tilde{A}=A+B_{2} K C_{2}$. The design problem leads to the following minimization problem:

$$
\min _{a, k, c} \quad\left\|\mathcal{H}_{\theta w}\right\|_{\infty}
$$

subject to: Condition (33)

$$
\begin{aligned}
& a_{l} \leq a \leq a_{h} \\
& k_{l} \leq k \leq k_{h} \\
& c_{l} \leq c \leq c_{h},
\end{aligned}
$$

where $\left[a_{l}, a_{h}\right],\left[k_{l}, k_{h}\right]$ and $\left[c_{l}, c_{h}\right]$ are the assigned proper ranges for $a, k$ and $c$ from the point of view of reliability and feasibility. A pioneering study on a combined wind-wave energy converter system using a variety of combinations of $a, k$ and $c$ is given in [20].

Owing to the constraint conditions, the optimization problem (35) cannot be analytically solved, for instance, by LMI approach. For a specified sea state, the following nu-

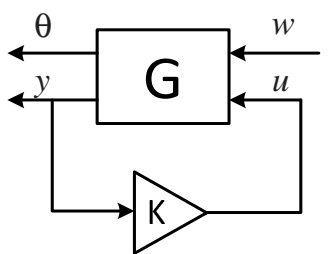

Fig. 3. Design of spring-damper systems is equal to determine the feedback controller $K$.

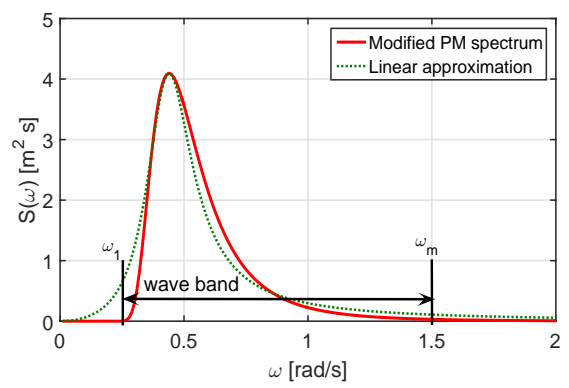

Fig. 4. Plot with solid line showing the modified Pierson-Moskowitz spectra for $H_{S}=4.5 \mathrm{~m}$ and $T_{z}=10 \mathrm{~s}$. The linear approximation by (28) is plotted with dotted line.

merical approach is proposed:

$$
\begin{aligned}
\min _{a, k, c} \quad \max _{i=1}^{m} & \left\|C_{1}\left(j \omega_{i} I-\tilde{A}\right)^{-1} B_{1}\right\| \\
\text { subject to: } & \text { Condition (33) } \\
& a_{l} \leq a \leq a_{h} \\
& k_{l} \leq k \leq k_{h} \\
& c_{l} \leq c \leq c_{h}
\end{aligned}
$$

where $\omega_{i}(i=1,2, \cdots, m)$ are the typical wave frequencies in wave band. It is not necessary to choose $\omega_{i}$ outside the wave band since there is little wave power there. A numerical example shown in Fig. 4 illustrates this concept. The optimization problem (36) can be solved by nonlinear programming solvers, such as Genetic Algorithm and Particle Swarm Optimization method [21].

\section{Numerical Example}

In this section, numerical examples are performed to verify the proposed design method. The dimensions of the semi-submersible are summarized in Table 1. Considering a fully developed sea with large depth, the modified PiersonMoskowitz (MPM) Spectrum is exploited to describe the sea state:

$$
S(\omega)=A \omega^{-5} \exp \left(-B \omega^{-4}\right)
$$

where $A$ and $B$ are parameters assigned by $A=\frac{4 \pi^{3} H_{s}^{2}}{T_{z}^{4}}$ and $B=\frac{16 \pi^{3}}{T_{z}^{4}}$ in which $H_{s}$ is the significant wave height and $T_{z}$ is the average zero-crossing wave period. A moderate sea 


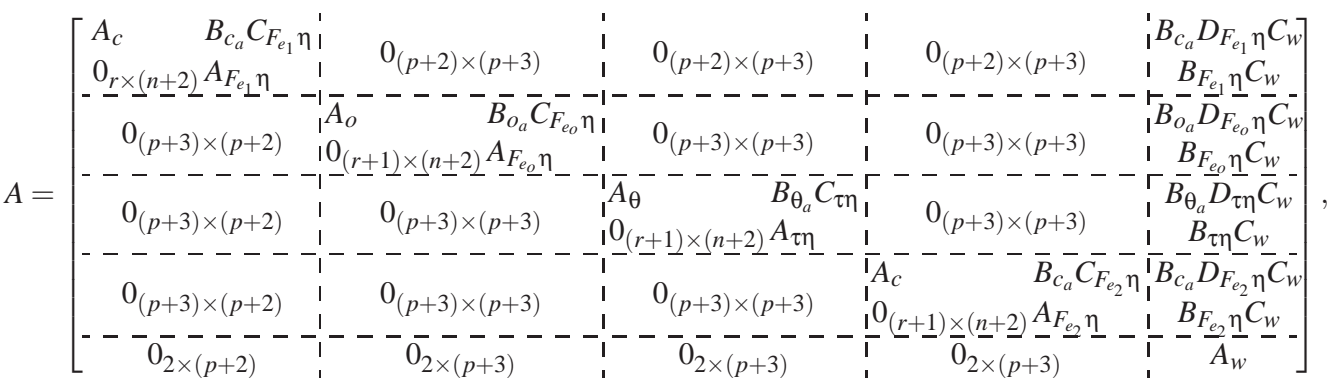

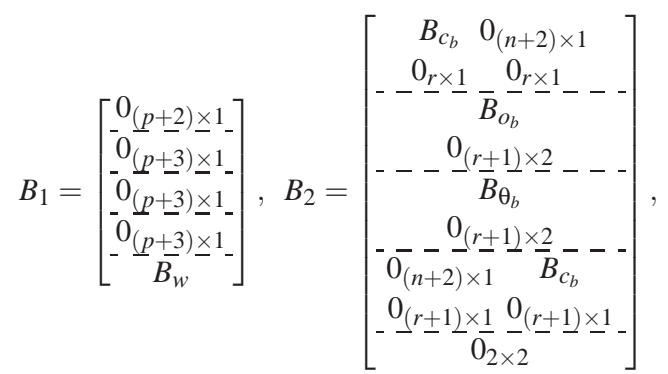

$$
\begin{aligned}
& C_{1}=\left[\begin{array}{lllll}
0_{1 \times(p+2)},{ }^{\prime} 0_{1 \times(p+3)},{ }^{\prime} 0_{1 \times n} & 1 & 0 & 0_{1 \times(r+1)},{ }^{\prime} 0_{1 \times(p+3)},{ }^{\prime} 0_{1 \times 2}
\end{array}\right] \text {, }
\end{aligned}
$$

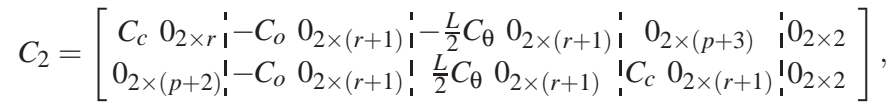

Table 1. Characteristics of floating platform

\begin{tabular}{lr}
\hline Property & Value \\
\hline distance between columns [m] & 60 \\
diameter of columns [m] & 10 \\
diameter $\times$ length of pontoon $[\mathrm{m} \times \mathrm{m}]$ & $10 \times 45$ \\
thickness $\times$ width of pillar $[\mathrm{m} \times \mathrm{m}]$ & $1 \times 10$ \\
draft [m] & 17 \\
submerged volume $\left[\mathrm{m}^{3}\right]$ & 6545 \\
total mass [kg] & $6,708,000$ \\
$\mathrm{Z}_{\mathrm{CoG}}[\mathrm{m}]$ & -9.5 \\
$\mathrm{Z}_{\mathrm{CoB}}[\mathrm{m}]$ & -10.4 \\
GM of main body [m] & 0.7 \\
GM of system (rigidly connected) $[\mathrm{m}]$ & 22.5 \\
\hline
\end{tabular}

state with the significant wave height as $H_{s}=4.5 \mathrm{~m}$ and the average zero-crossing wave period as $T_{z}=10 \mathrm{~s}$ is applied. The wave spectra is shown in Fig. 4. It can be observed that the wave band is in the interval $[0.24,1.5] \mathrm{rad} / \mathrm{s}$. The linear approximation by transfer function (28) is also plotted in the figure by using curve fitting method, and the resulting wave intensity is $\sigma=2.02$ and damping coefficient is $\lambda=0.256$.

Hydrodynamic codes based on constant panel method [22] are applied to compute the frequency-dependent added mass, potential damping and wave excitation coefficients in
Table 2. Added mass coefficients at infinite frequency

\begin{tabular}{lr}
\hline Variable & Value \\
\hline Heave of column $[\mathrm{kg}]$ & $2.638 \times 10^{5}$ \\
Heave of main body $[\mathrm{kg}]$ & $3.117 \times 10^{6}$ \\
Pitch of main body $\left[\mathrm{kg} \cdot \mathrm{m}^{2}\right]$ & $5.279 \times 10^{8}$ \\
\hline
\end{tabular}

frequency domain. The added mass at infinite frequency are shown in Table. 2. Owing to the limitation of our research codes on calculating multibody structures, hydrodynamic interaction between the different bodies is neglected. Whilst the inclusion of the hydrodynamic interaction would affect the final results, the proposed method applied here would still be valid once the models is updated. The toolbox developed by T. Perez and T. I. Fossen [23] are applied to identify the state-space models of the memory functions of the main body and the columns, and the results are shown in Fig. 5. It can be observed that the linear approximation models fit well with the theory, especially in the wave band. Characteristics of wave excitation loads on the main body and the columns, and the comparison with linear models are shown in Fig. 6. Owing to the non-causality and nonlinearity, the phases are not coincident with theory. However, it is sufficient for our design purpose. In addition, the wave propagation periods $T_{1}$ and $T_{2}$ are $T_{1}=2.69 \mathrm{~s}$ and $T_{2}=5.38 \mathrm{~s}$ calculated from the dominating wave frequency $\omega_{0}$.

The linear coefficient of $J_{p}$ to $m_{p}$ in (10) is set as $\gamma=$ 625. The range of $a$ is set as $a \in[0.03,0.12]$ according to the prior numerical study in [20] that the optimal $a$ is close to 



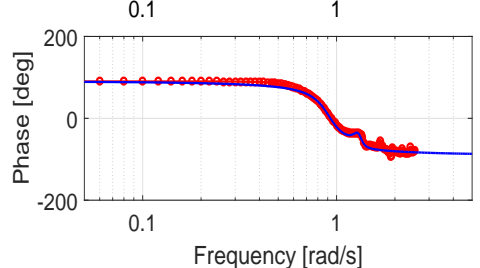

(a) Memory function of main body in pitching direction $K_{\theta_{r}}$.

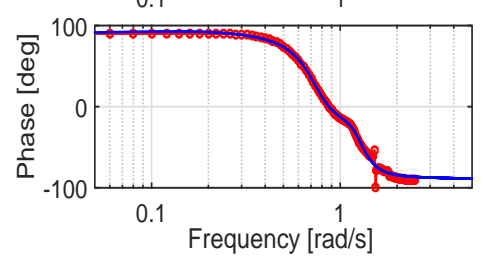

(b) Memory function of main body in heaving direction $K_{h_{r}}$.

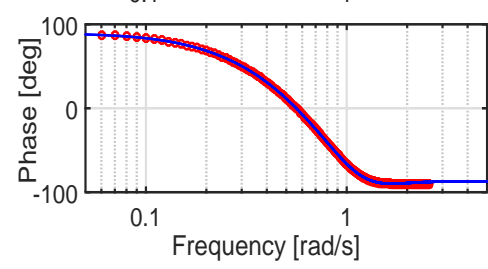

(c) Memory function of column in heaving direction $K_{c_{r}}$.

Fig. 5. Memory functions computed from potential theory (solid lines with circle markers) and their linear approximation (solid lines).


(a) Wave excitation loads to main body in pitching direction.
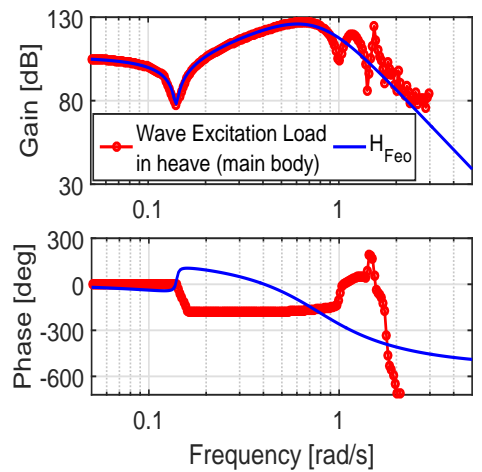

(b) Wave excitation loads to main body in heaving direction.
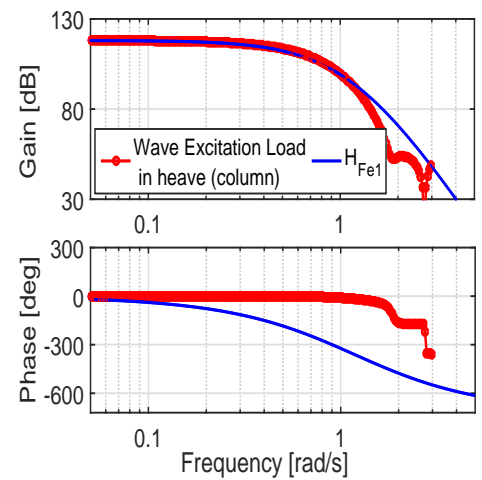

(c) Wave excitation loads to column in heaving direction.

Fig. 6. Wave excitation loads computed from potential theory (solid lines with circle markers) and their linear approximation (solid lines).

five percent. The term $k$ is selected in $k \in[5,50] \times 10^{4} \mathrm{~N} / \mathrm{m}$ to keep the relative displacements between the columns and the main body smaller than $6 \mathrm{~m}$, which is $10 \%$ of the distance of two columns. The term $c$ is properly selected as $c \in[0.1,30] \times 10^{5} \mathrm{Ns} / \mathrm{m}$. The typical frequencies $\omega_{1}, \cdots, \omega_{m}$ with $m=100$ to equally divide the wave band are applied in (36). The Particle Swarm Optimization Algorithm provided by Matlab ${ }^{\circledR}$ is applied for solving the constrained optimization problem (36). The default settings that the number of particles is 100 and the maximum number of iterations is 600 are employed. Iterations end when the relative change in best objective function value over the last iteration is less than $1.0 \times 10^{-6}$. In this study, a solution is obtained after 21 iterations, and the computational time is $28.4 \mathrm{~s}$ by Intel ${ }^{\circledR}$ Core i7-4790 CPU @ $3.60 \mathrm{GHz} \times 2$ with 8 GB of memory.
The optimum coefficients are

$$
\begin{aligned}
a_{\mathrm{opt}} & =0.03 \\
k_{\mathrm{opt}} & =5.00 \times 10^{4}[\mathrm{~N} / \mathrm{m}], \\
c_{\mathrm{opt}} & =9.52 \times 10^{5}[\mathrm{~N} \mathrm{~s} / \mathrm{m}] .
\end{aligned}
$$

From the results, it is observed that the optimal spring coefficient $k$ hits the lower bound of the given range. This means the stronger the stiffness of spring is, the larger pitch motion would be excited. The perspective is also illustrated in Fig. 7(a) when $k$ varies in its range with $c=c_{\text {opt }}$ and $a=a_{\text {opt }}$. In the figure, the Bode magnitude plot of the transfer function $\mathcal{H}_{\theta w}(j \omega)$ with the magnitude in decibels by $20 \log _{10}\left|\mathcal{H}_{\theta w}\right|$ is exploited. It is shown that the amplitude of pitch angle increases when $k$ varies toward the upper bound of the given range. Besides, $a$ also hit its lower bound of the range. The comparison of the case $a=a_{\mathrm{opt}}$ with other $a$ are shown in Fig. 7(c). The effect of $a$ on pitching motion will be dis- 


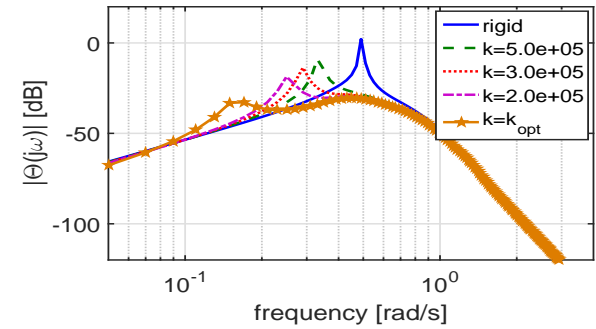

(a) Characteristics when $c=c_{\mathrm{opt}}$ and $a=a_{\mathrm{opt}}$.

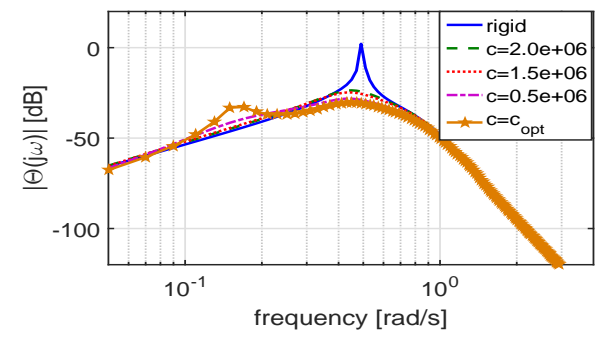

(b) Characteristics when $k=k_{\mathrm{opt}}$ and $a=a_{\mathrm{opt}}$.



(c) Characteristics when $c=c_{\mathrm{opt}}$ and $k=k_{\mathrm{opt}}$.

Fig. 7. Frequency characteristics when varying $k, c$ and $a$ from the optimal values. The magnitude is in decibels by $20 \log _{10}|\theta(j \omega)|$.

cussed further in our future work. Fig. 7(b) shows that the smallest pitching motion can be obtained by $c=c_{\text {opt }}$.

In addition, numerical results in the time domain using wave excitation loads calculated by potential theory are shown in Fig. 8. The calculated optimal case is plotted in solid line with star marker in the figure. The maximum pitching angles as well as the root-mean-square values of different cases are given in Table 3. It is also obtained that the calculated optimum coefficients can achieve the smallest inclination. From these results, we can also obtained that the model errors caused by the linearizion using (23-26) (especially in the phase) do not affect much on the performance of the design method.

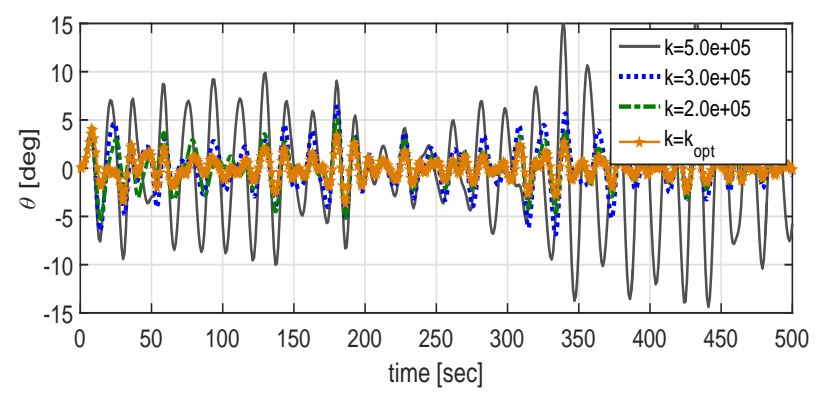

(a) Pitch motion when $c=c_{\mathrm{opt}}$ and $a=a_{\mathrm{opt}}$.

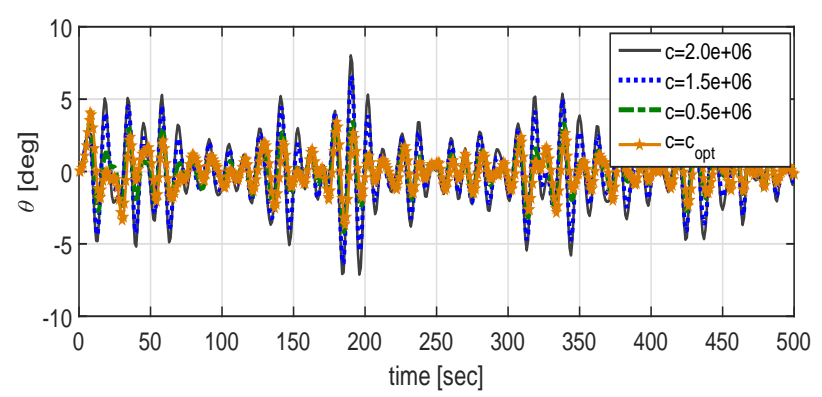

(b) Pitch motion when $k=k_{\mathrm{opt}}$ and $a=a_{\mathrm{opt}}$.

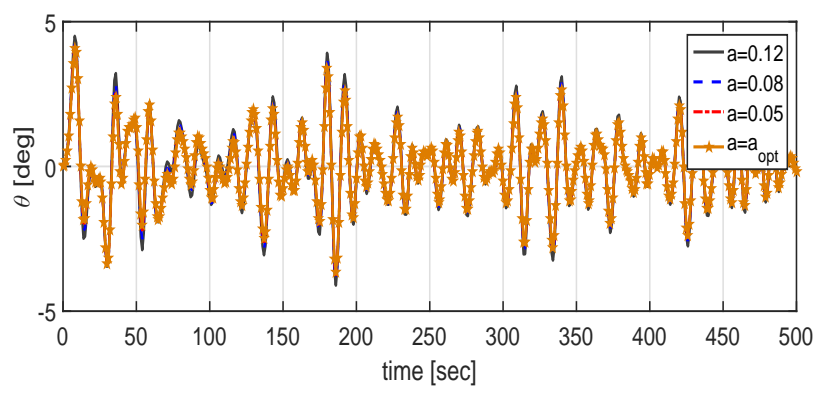

(c) Pitch motion when $c=c_{\mathrm{opt}}$ and $k=k_{\mathrm{opt}}$.

Fig. 8. Numerical results when varying $k, c$ and $a$.

\section{Conclusion}

In this paper, a design method of a semi-submersible with suspension structures to suppress the pitch motion was presented. Linear models were applied to model the radiation loads and wave excitation loads so that the whole system can be expressed by a state-space model. The design of the passive suspension system leads to a mathematically tractable problem from the viewpoint of feedback control. It was verified by numerical examples that the optimum suspension coefficients could be obtained by solving a constrained optimization problem. The proposed method can be applied to design the WEC for a combined wave-wind energy system considering the effect of WEC to pitch motion of FOWT. 
Table 3. Comparison of the pitching angles

\begin{tabular}{|c|c|c|c|}
\hline \multicolumn{2}{|c|}{ Case } & max. value & RMS value \\
\hline \multicolumn{2}{|c|}{$\begin{array}{l}k=k_{\mathrm{opt}}, c=c_{\mathrm{opt}} \text { and } \\
a=a_{\mathrm{opt}}\end{array}$} & $4.08^{\circ}$ & $1.15^{\circ}$ \\
\hline \multirow{3}{*}{$\begin{array}{l}c=c_{\mathrm{opt}} \\
a=a_{\mathrm{opt}}\end{array}$} & $k=5.0 \times 10^{5}$ & $15.82^{\circ}$ & $6.21^{\circ}$ \\
\hline & $k=3.0 \times 10^{5}$ & $6.92^{\circ}$ & $2.23^{\circ}$ \\
\hline & $k=2.0 \times 10^{5}$ & $5.15^{\circ}$ & $1.74^{\circ}$ \\
\hline \multirow{3}{*}{$\begin{array}{l}k=k_{\mathrm{opt}} \\
a=a_{\mathrm{opt}}\end{array}$} & $c=2.0 \times 10^{6}$ & $8.02^{\circ}$ & $2.44^{\circ}$ \\
\hline & $c=1.5 \times 10^{6}$ & $6.58^{\circ}$ & $2.03^{\circ}$ \\
\hline & $c=5.0 \times 10^{5}$ & $4.26^{\circ}$ & $1.27^{\circ}$ \\
\hline \multirow{3}{*}{$\begin{array}{l}k=k_{\mathrm{opt}} \\
c=c_{\mathrm{opt}}\end{array}$} & $a=0.12$ & $4.50^{\circ}$ & $1.32^{\circ}$ \\
\hline & $a=0.08$ & $4.31^{\mathrm{o}}$ & $1.25^{\circ}$ \\
\hline & $a=0.05$ & $4.17^{\circ}$ & $1.19^{\circ}$ \\
\hline
\end{tabular}

\section{Acknowledgements}

This research was partially supported by JSPS KAKENHI Grant Number 15H06472.

\section{References}

[1] Ishihara, T., Phuc, P., Sukegawa, H., Shimada, K., and Ohyama, T., 2007. "A study on the dynamic response of a semi-submersible floating offshore wind turbine system part 1: A water tank test". In Proceedings of the 12th International Conference on Wind Engineering, pp. 2511-2518.

[2] Henderson, A. R., and Patel, M. H., 2003. "On the modelling of a floating offshore wind turbine". Wind Energy, 6(1), pp. 53-86.

[3] Faltinsen, O., 1993. Sea Loads on Ships and Offshore Structures. Cambridge University Press, Cambridge.

[4] Sato, A., and Suzuki, H., 2006. "Effect of motion of floating platform on the strength design of floating wind turbine". In Proceedings of 28th Symposium on Wind Energy Utilization, pp. 192-195. in Japanese.

[5] Suzuki, H., and Sato, A., 2007. "Load on turbine blade induced by motion of floating platform and design requirement for the platform". In Proceedings of the 26th International Conference on Offshore Mechanics and Arctic Engineering, pp. 519-525.

[6] Huijs, F., Mikx, J., Savenije, F., and de Ridder, E.-J., 2013. "Integrated design of floater, mooring and control system for a semi-submersible floating wind turbine”. In EWEA Offshore 2013 Vienna.

[7] Roddier, D., Cermelli, C., Aubault, A., and Weinstein, A., 2010. "Windfloat: A floating foundation for offshore wind turbine". Journal of Renewable and Sustainable Energy, 2, p. 033104.

[8] Karimirad, M., and Michailides, C., 2015. "V-shaped semisubmersible offshore wind turbine: An alternative concept for offshore wind technology". Renewable Energy, 83, pp. 126-143.
[9] Aubault, A., Cermelli, C., and Roddier, D., 2006. "Structural design of a semi-submersible platform with water-entrapment plates based on a time-domain hydrodynamic algorithm coupled with finite-elements". In Proceedings of 6th International Offshore and Polar Engineering Conference, pp. 187-194.

[10] Zhu, H., Ou, J., and Zhai, G., 2012. "Conceptual design of a deep draft semi-submersible platform with a movable heave-plate". Journal of Ocean University of China, 11(1), pp. 7-12.

[11] Roddier, D., and Cermelli, C., 2013. Column-stabilized offshore platform with water-entrapment plates and asymmetric mooring system for support of offshore wind turbines. patent, US008471396B2.

[12] Chandrasekaran, S., Raphel, D., and Shree, S., 2014. "Deep ocean wave energy systems (dowes): experimental investigations". Journal of Naval Architecture and Marine Engineering, 11(2), pp. 139-146.

[13] Perez, C., Greaves, D., and Iglesias, G., 2015. "A review of combined wave and offshore wind energy". Renewable and Sustainable Energy Reviews, 42, pp. 141153.

[14] Perez, C., and Iglesias, G., 2012. "Integration of wave energy converters and offshore windmills". In Proceedings of the fourth international conference on ocean energy (ICOE).

[15] Kral, R., and Kreuzer, E., 1999. "Multibody systems and fluid-structure interactions with application to floating structures". Multibody System Dynamics, 3(1), pp. 65-83.

[16] Cummins, W. E., 1962. The impulse response function and ship motions. Tech. Rep. 47, Schiffstechnik.

[17] Perez, T., and Fossen, T., 2009. Identification of dynamic models of marine structures from frequencydomain data enforcing model structure and parameter constraints. Tech. rep., ARC Centre of Excellence for Complex Dynamic Systems and Control.

[18] Yu, Z., and Falnes, J., 1995. "State-space modelling of a vertical cylinder in heave". Applied Ocean Research, 17, pp. 265-275.

[19] Fossen, T. I., 2011. Handbook of Marine Craft Hydrodynamics and Motion Control. John Wiley \& Sons, Ltd, UK.

[20] Borg, M., Collu, M., and Brennan, F. P., 2013. "Use of a wave energy converter as a motion suppression device for floating wind turbines". Energy Procedia, 35, pp. 223-233.

[21] Kennedy, J., and Eberhart, R., 1995. "Particle swarm optimization". In Proc. IEEE Int. Conf. Neural Networks, Vol. 4, pp. 1942-1948.

[22] Liu, Y., Iwashita, H., and Hu, C., 2015. "A calculation method for finite depth free-surface green function". International Journal of Naval Architecture and Ocean Engineering, 7(2), pp. 375-389.

[23] Perez, T., and Fossen, T., 2009. "A matlab toolbox for parametric identification of radiation-force models of ships and offshore structures". Modeling, Identification and Control, 30(1), pp. 1-15. 